\title{
Health-related quality of life and unmet needs in patients with primary ciliary dyskinesia
}

\author{
M. Pifferi*, A. Bush", M. Di Cicco*, U. Pradal", V. Ragazzo*, \\ P. Macchia* and A.L. Boner ${ }^{+}$
}

ABSTRACT: Few studies have evaluated the quality of life of patients with primary ciliary dyskinesia (PCD). We sought to determine the health impact of the disease as well as the unmet needs in a large group of patients.

Questionnaires were either posted or e-mailed to known patients with PCD and published online. Questionnaires included the St George's Respiratory Questionnaire, the Medical Outcomes Study Short Form-36 and a questionnaire that we produced to obtain information on age of diagnosis, symptoms and likely PCD-specific problems of these patients.

78 subjects ( $96 \%$ of those invited) answered all the questionnaires. Patients were diagnosed at a mean age of 9.4 yrs. Progressive worsening of the disease was observed and adherence to physiotherapy was found to be poor, particularly in adolescents and adults. Patients with the highest treatment burden had a worse quality of life. Over time patients become progressively less interested in treating their disease and adherence to treatment modalities decreases.

PCD is associated with a progressive and continuous impact on the physical and mental health of the patients. Earlier identification of the patients and better strategies aimed at improving compliance with care are urgently needed.

KEYWORDS: Medical Outcomes Study Short Form-36, primary ciliary dyskinesia, quality of life, St George's Respiratory Questionnaire, unmet needs

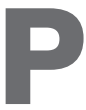

rimary ciliary dyskinesia (PCD) is an uncommon and difficult to diagnose disease. It is a phenotypically and genetically heterogeneous disorder due to congenital abnormalities in structure and function of respiratory cilia $[1,2]$. Typically, impairment of mucociliary clearance leads to recurrent infections of the upper and lower respiratory tract with chronic symptoms, which include a daily productive cough. Since the diagnosis of PCD is frequently delayed, lung damage and bronchiectasis may occur before treatment is initiated [3], and eventually a significant deterioration in respiratory function may occur. Therefore, early diagnosis is desirable so appropriate treatment may be started (physiotherapy and antibiotics). It is hoped this would prevent the most serious long-term consequences. Indeed, PCD patients diagnosed late appear to have by worse lung function $[4,5]$.

Much research on PCD focuses on the genetics and pathophysiology of the disease [6]. To date, only one study has evaluated the effects of illness on the overall health status of PCD patients [7]. In particular, this study assessed the pattern of symptoms, describing their variability and development over the life span, and measured the impact of the disease on the lifestyle and mental health of those patients living in the UK. Diseasespecific studies are important because in PCD patients there is prominent involvement of both upper and lower airways. Specifically, and unlike in most people who have chronic suppurative lung disease, PCD patients experience the early onset of significant chronic rhinitis, otitis media and chronic rhinosinusitis. This is important since upper airway disease causes significant morbidity [8, 9]. Furthermore, since prognosis is better in PCD patients compared to that observed in subjects with cystic fibrosis (CF), PCD is in danger of being trivialised.

The aim of our study was to assess the impact of PCD on health-related quality of life (HRQoL) in
AFFILIATIONS

*Dept of Paediatrics, University of Pisa, Pisa

- Cystic Fibrosis Center, Azienda Ospedaliera Verona, and ${ }^{+}$Dept of Paediatrics, University of Verona, Verona, Italy.

" Imperial School of Medicine at the National Heart and Lung Institute, London, UK.

CORRESPONDENCE

M. Pifferi

Dept of Paediatrics

University of Pisa; Via Roma 67 56126 Pisa

Italy

E-mail: m.pifferi@med.unipi.it

Received:

March 302009

Accepted after revision:

Aug 272009

First published online:

Sept 242009 
Italian patients. Moreover, since a delay in recognising the disease and/or its inappropriate management may lead to adverse consequences for patients with PCD, the second aim of the study was to identify the unmet needs of the patients and the potential diagnostic and therapeutic pitfalls.

\section{MATERIALS AND METHODS}

In December 2007, questionnaires were posted or e-mailed to patients with a diagnosis of PCD made in the Dept of Paediatrics (University Hospital of Pisa, Pisa, Italy), in the Cystic Fibrosis Center (Hospital of Verona, Verona, Italy), in the Dept of Paediatrics (University of Verona, Verona) or to patients who contacted us after the publication of the HRQoL survey project on our website [10]. The questionnaires were returned before May 30, 2008, when the survey was concluded. All the patients seen at tertiary centres were diagnosed with electron microscopic evaluation of ciliary structure and orientation. Any patient who had not previously attended a specialist centre was seen in Pisa to confirm the diagnosis. Ciliary beat frequency measurements and ciliary beat pattern analysis were performed in all the patients followed in Pisa.

There is no validated, disease-specific instrument to assess quality of life in PCD. However, the St George's Respiratory Questionnaire (SGRQ) has previously been validated in patients with bronchiectasis [11], and is available in a validated translated Italian [12]. Due to this we applied the SGRQ, together with an Italian version of the Medical Outcomes Study Short Form-36 (SF-36) [13]. Furthermore, we devised a questionnaire on the clinical course of the disease plus the investigation and management, which includes a question on the patient's perception of quality of life after receiving the diagnosis (see supplementary data). As previously suggested [7], separate versions of the questionnaires with minor rewording changes were provided for adults, co-operative older children and for younger, non co-operative children. We attached a letter suggesting the parents helped their children in answering the questions, or answered the questions themselves when children were too young to collaborate. We asked parents to report if the questionnaire was completed by the patient alone, if the child was helped by the parents or if only the parents answered the questions.

The SGRQ is a self-administered HRQoL measure containing 50 items and 76 weighted responses divided into three components: symptoms, activity and impacts [14]. The symptoms component comprises of eight items concerning the level of symptoms, including frequency of cough, sputum production, wheeze, breathlessness, and the duration and frequency of breathlessness or wheeze. The activity component (16 items) is concerned with physical activities that either cause or are limited by breathlessness. The impacts component (26 items) covers a range of aspects concerning social functioning and psychological disturbances resulting from airways disease. Scores ranging from 0 to 100 are calculated for each component, as well as a total score which summarises the responses to all items. A zero score indicates no impairment of quality of life. The questionnaire can be completed in $\sim 10 \mathrm{~min}$.

The SF-36 questionnaire is a self-administered questionnaire containing 36 items which provide eight scales, four of which relate to physical health: physical functioning, role physical, bodily pain and general health. The remaining four scales are related to mental health: vitality, social functioning, role emotional and mental health.

Each scale is scored from 0 to 100 . A score of 100 in physical functioning, role physical, bodily pain, social functioning and role emotional indicates absence of limitations or disability, while in general health, mental health and vitality the best health corresponds to a score of 50 . These eight scales provide two summary scores: the Physical Component Summary (PCS) and the Mental Component Summary (MCS), in which a normal score is $50 \pm 10$. The normal value is 50 and diminishing scores indicate worsening conditions. The SF-36 questionnaire can be completed in only $5 \mathrm{~min}$. Separate age and sex-related norms for the SF-36 questionnaire reported by APOLONE and co-workers $[13,15]$ in Italians aged $>18$ yrs, and reference values obtained in 30 healthy age- and sex-matched (15 males and 15 females, aged $8.6 \pm 4.4$ yrs) children were used for comparison.

We used a study-specific questionnaire on PCD/Kartagener Syndrome comprising of 15 questions relating to diagnosis, clinical features, follow-up, therapy and the presence of other PCD patients within the family (see supplementary data). The questionnaire can be completed within $5 \mathrm{~min}$.

Informed consent for the survey was obtained from adult patients or from parents of children with PCD and the study protocol was approved by the Hospital Ethical Committee of Pisa.

\section{Statistical analyses}

Baseline variables are presented as group mean \pm SD. Differences between means and distributions were evaluated by the two-tailed unpaired t-test. The Chi-squared test was used to evaluate the association between the presence of mirror image arrangement and diagnosis of PCD.

The statistical significance of correlations between each component of the SGRQ and SF-36 questionnaire (including the total score, which summarises the responses to all items), and other variables, such as the age and features of the patients gathered by the questionnaire on PCD/Kartagener Syndrome, were examined using the Pearson correlation test and Spearman rank signed test. Multiple linear regression analyses were conducted to evaluate the associations between the dependent variables (SGRQ symptoms, SGRQ activity, SGRQ impacts, SF-36 PCS and SF-36 MCS) and the independent variable (age, age at diagnosis and time since diagnosis (calculated as current age minus age at diagnosis)). A p-value $<0.05$ was considered statistically significant.

Since the SGRQ and SF-36 are questionnaires designed for adults, sub-analyses were performed: age $\geqslant 18$ yrs versus $<18$ yrs, and either including or excluding children requiring any assistance in completing the SGRQ and SF-36 questionnaires.

All statistical calculations were performed using SPSS version 14.0 (SPSS Inc., Chicago, IL, USA) for personal computers.

\section{RESULTS}

\section{Participants in the survey}

The questionnaires and the covering letter were sent to 81 addresses. 78 (96\%) subjects completed all the three questionnaires. 58 patients were followed in the tertiary centres of Pisa 
$(n=45)$ and Verona $(n=13)$ and 20 were recruited through the website. The 20 patients recruited through the website were members of the Italian Association Kartagener/PCD and Italian Association Dyskinesia-Kartagener ONLUS. All the patients followed in the tertiary centres were invited to participate irrespective of age and severity of the disease. The age of the participants (34 males and 44 females) ranged from 1.7 to 48.5 yrs (mean \pm SD $21.4 \pm 12.9$ yrs). Female respondents were slightly, although not significantly $(p=0.08)$, older (mean $\pm S D$ $23.6 \pm 13.1 \mathrm{yrs}$ ) than males (mean \pm SD $18.5 \pm 12.3 \mathrm{yrs}$ ). There were 35 children aged $<18$ yrs (mean age \pm SD $9.04 \pm 3.4$ yrs). Parents reported helping their children in answering questions in three subjects (aged 5.5, 5.9 and 6.5 yrs, respectively) and being the sole respondent in the four youngest patients (aged $1.7,2.4,3.9$ and 4.9 yrs, respectively). All the remaining 28 children (12 males; mean \pm SD $10.2 \pm 2.6$ yrs, range $7.2-18$ yrs), were reported as completing the questionnaires unassisted and were included separately in the sub-analysis. The mean \pm SD age of the 43 adult patients was $31.4 \pm 8.2$ yrs.

\section{SGRQ}

The three sub-scales of the SGRQ all correlated highly with one another (symptoms with activity: $\mathrm{r}=0.600, \mathrm{p}<0.001$; symptoms with impacts: $\mathrm{r}=0.624, \mathrm{p}<0.001$; and activity with impacts; $\mathrm{r}=0.705, \mathrm{p}<0.001$ ). This remained true for the 43 patients aged $\geqslant 18$ yrs and in the younger subjects whether or not the seven children who were unable to complete the instruments themselves were considered. Considering the whole study population, all three sub-scales correlated significantly with age (symptoms: $\mathrm{r}=0.285, \mathrm{p}=0.01$; activity: $\mathrm{r}=0.295, \mathrm{p}=0.009$; and impacts: $r=0.539, \mathrm{p}<0.001$ ), while no correlation was found with the age of the patients in any subgroup.

Cough, on almost all days of the week in the last 12 months, was the most frequently reported symptom (48.7\% of patients) regardless of age, together with excessive phlegm $(57.7 \%$ of patients), which significantly increased with age $(\mathrm{p}<0.001)$. This was true also in the adult subgroup for cough $(\mathrm{p}=0.028)$ but not for phlegm. In patients aged $<18$ yrs (excluding the seven subjects who needed assistance in completing the questionnaire), the increase in cough and phlegm with age was still significant ( $p=0.05$ for both). Furthermore, in the whole study population, a significant correlation was found between time since diagnosis and impacts sub-scale (Spearman $\mathrm{p}<0.0001, \mathrm{r}=0.403$; Pearson $\mathrm{p}=0.01, \mathrm{r}=0.31$ ) and for the activity sub-scale (Spearman $\mathrm{p}=0.001 \mathrm{r}=0.368$; Pearson $p<0.0001, r=0.37$ ), while no significant correlation was found for symptoms sub-scale. In both the adult patients and those aged $<18$ yrs (excluding the seven children who needed assistance) there was no significant correlation between time since diagnosis and any of the three sub-scales.

Breathlessness, which was mostly an issue at the time of respiratory infections $(37.2 \%)$, worsened with age in the whole study population $(\mathrm{p}<0.001)$. The correlation with age is lost if the patients aged $<18$ yrs are excluded. However, the scores were significantly worse in adults ( $\geqslant 18$ yrs) compared with younger patients $(<18 \mathrm{yrs})$ for symptoms, impacts and activity: $47.2 \pm 23.1$ versus $36.01 \pm 16.5(\mathrm{p}=0.009), \quad 30.5 \pm 20.3$ versus $12.8 \pm 9.8(\mathrm{p}=0.00001)$, and $29.1 \pm 24.5$ versus $16.7 \pm 18.7$ $(p=0.008)$, respectively. This was true even if the seven children who required assistance were excluded: $47.2 \pm 23.1$ versus $37.1 \pm 16.7 \quad(p=0.025), \quad 30.5 \pm 20.3$ versus $14.5 \pm 10.3$ $(\mathrm{p}=0.0001)$, and $29.1 \pm 24.5$ versus $18.4 \pm 16.6(\mathrm{p}=0.02)$, respectively. This suggests that deterioration of symptoms may initially be progressive, but that subsequently there is a stabilisation and an adaptation or a reduced perception of worsening by the patients. There were no significant sex differences in the scores.

\section{The SF-36 measures of health status}

We observed a decline in PCS and MCS scores (and also in each sub-scale) in relation to age in the PCD patients. However, the negative correlation was only significant for MCS $(p<0.0001, r=-0.447)$. For both scores the decline was significantly greater than that reported with normal ageing in the healthy Italian population [13, 15]: age 0-18 yrs (whether the seven children who required assistance were included or not) PCS $p<0.001$ and MCS $p=0.02$ (without the seven children PCS $\mathrm{p}<0.001$ and MCS $\mathrm{p}=0.001)$; age $18-24$ yrs: PCS $p=0.001$ and MCS $p$-value not significant; age $25-34$ yrs: PCS $\mathrm{p}<0.001$ and MCS $\mathrm{p}=0.04$; age 35-44 yrs: PCS $\mathrm{p}=0.02$ and MCS p-value not significant; age 45-54 yrs: PCS p-value not significant and MCS $p=0.003$. Again, no sex differences were found among PCD patients. There was no correlation between PCS and MCS and age in adults aged $\geqslant 18$ yrs, or in younger subjects, excluding the seven children who required assistance with the questionnaire.

\section{Questionnaire on PCD/Kartagener syndrome}

The median (range) age at diagnosis was 7.7 (0-44) yrs (mean $\pm \mathrm{SD} 9.4 \pm 10.4 \mathrm{yrs}$ ); there was a trend for earlier diagnosis $(p=0.08)$ for PCD with mirror image arrangement (situs inversus: mean $\pm \mathrm{SD} 7.9 \pm 9.7 \mathrm{yrs}$, median $5 \mathrm{yrs}$ ) than in PCD with usual organ arrangement (situs solitus: mean \pm SD $12.4 \pm 11.3 \mathrm{yrs}$, median $8 \mathrm{yrs}$ ). Thus, the presence of situs inversus may result in earlier diagnosis. Situs solitus is significantly correlated with late diagnosis in the subgroup of adult patients $(p=0.013)$. However, the scatter in both groups was very large, indicating that, in part, the older patients had only been diagnosed relatively recently (12 patients were diagnosed when aged $>20$ yrs $(29.6 \pm 8.0$ yrs $)$. There was a non-significant $(\mathrm{p}=0.1)$ trend for later diagnosis in females $(11.1 \pm 11.8$ yrs versus $7.4 \pm 7.9$ yrs $)$ than in males.

Age at diagnosis in relation to symptom scores was correlated with the responses acquired from the SGRQ and SF-36. Table 1 shows the regression of scores upon age, age at diagnosis and time since diagnosis for the whole study population. Age at diagnosis was significantly positively correlated with SGRQ symptoms, activity and impacts suggesting that older age at diagnosis results in worse effects on symptoms, activity and impacts, and also that mental health is worse. Furthermore, the significant positive correlation between time since diagnosis and SGRQ symptoms and impacts scores suggest that treatment is only partially effective or that adherence to treatment is progressively poorer, or both. Other than for symptoms, this remained true even when the seven children who required assistance completing the questionnaire were excluded (see table in supplementary data).

Reduced compliance with treatment is also suggested by the negative correlations between SF-36 MCS and both age at diagnosis and time since diagnosis. A reduction in SF-36 MCS 
TABLE 1 Multiple linear regression analysis on dependent and independent variables

\begin{tabular}{|c|c|c|c|c|c|c|}
\hline \multirow[t]{2}{*}{ Dependent variable } & \multicolumn{6}{|c|}{ Independent variables } \\
\hline & \multicolumn{2}{|c|}{ Age yrs } & \multicolumn{2}{|c|}{ Age at diagnosis yrs } & \multicolumn{2}{|c|}{ Time since diagnosis yrs } \\
\hline SGRQ symptoms & NS & NS & 0.243 & 0.040 & 0.242 & 0.041 \\
\hline SF-36 PCS & NS & NS & NS & NS & NS & NS \\
\hline SF-36 MCS & NS & NS & -0.388 & 0.001 & -0.351 & 0.002 \\
\hline
\end{tabular}

SGRQ: St George's Respiratory Questionnaire; SF-36: Short Form-36; PCS: physical component summary; MCS: mental component summary; NS: not significant. \# regression coefficient.

over time is a marker of progressive deterioration of the mental component of the patients. It may be that with time patients give-up and become fatalistic about the disease. This is also suggested by the fact that in the older group there is only a significant correlation with activity sub-scale $(\mathrm{p}=0.04)$, but further investigation is needed to confirm this. Moreover, in the whole study population, both age and age at diagnosis correlated with either the presence of sinusitis $(p<0.001$ and $\mathrm{p}=0.006$, respectively) and bronchiectasis $(\mathrm{p}<0.001$ and $\mathrm{p}=0.003$, respectively). The latter suggests that these clinical presentations are important diagnostic clues. In the sub-group of the 28 children who filled in the questionnaire without assistance, the presence of sinusitis correlated both with time since diagnosis $(p=0.008)$ and with age at diagnosis $(p=0.001)$. Furthermore, in the adult population the prevalence of sinusitis correlated with time since diagnosis $(p=0.033)$. In this group there is no correlation between bronchiectasis and age at diagnosis and time since diagnosis, suggesting that bronchiectasis may be an early event in this disease. This is further confirmed by the lack of any correlation when the 28 children who completed the questionnaire are considered separately. In addition, in the whole study population, age and age at diagnosis positively correlated with breathlessness $(\mathrm{p}<0.001$ and $\mathrm{p}<0.001$, respectively), which was also observed for age at diagnosis in the adults $(\mathrm{p}=0.004)$, but not in the 28 children who completed the questionnaire unassisted. This suggests that breathlessness is a late event.

\section{Presence of mirror image arrangement}

$50(64.1 \%)$ out of the 78 subjects reported mirror image arrangement, one $(1.3 \%)$ subject self-reported isolated dextrocardia and the remaining $27(34.6 \%)$ patients reported usual organ arrangement. There was no sex difference in the prevalence of mirror image arrangement (males $64.7 \%$ and females $63.6 \%$ ). There were no significant differences in respiratory symptoms and health status between PCD patients with and without mirror image arrangement. However, it was interesting that there were significantly more patients with situs inversus than situs solitus (Chi-squared 6.87, $\mathrm{p}<0.05$ ) suggesting that there is a pool of undiagnosed patients with situs solitus.

\section{Other clinical findings}

The prevalence of different clinical features is shown in figure 1. Three patients (two with mirror image arrangement) had congenital heart disease (ventricular septal defect, or rightsided partial anomalous pulmonary venous drainage with atrial septal defect).

\section{Diagnostic procedures and follow-up}

A variable pattern of diagnostic procedures have been used (fig. 2). In particular, two patients who answered the questionnaires through the website had a final diagnosis based only on clinical features (mirror image arrangement plus bronchiectasis and chronic sinusitis). These two patients were subsequently evaluated by us with transmission electron microscopy and ciliary motion analysis, confirming the presence of PCD (both patients had absent dynein arms). Moreover, it is noteworthy that noninvasive procedures, such as audiometry and lung function

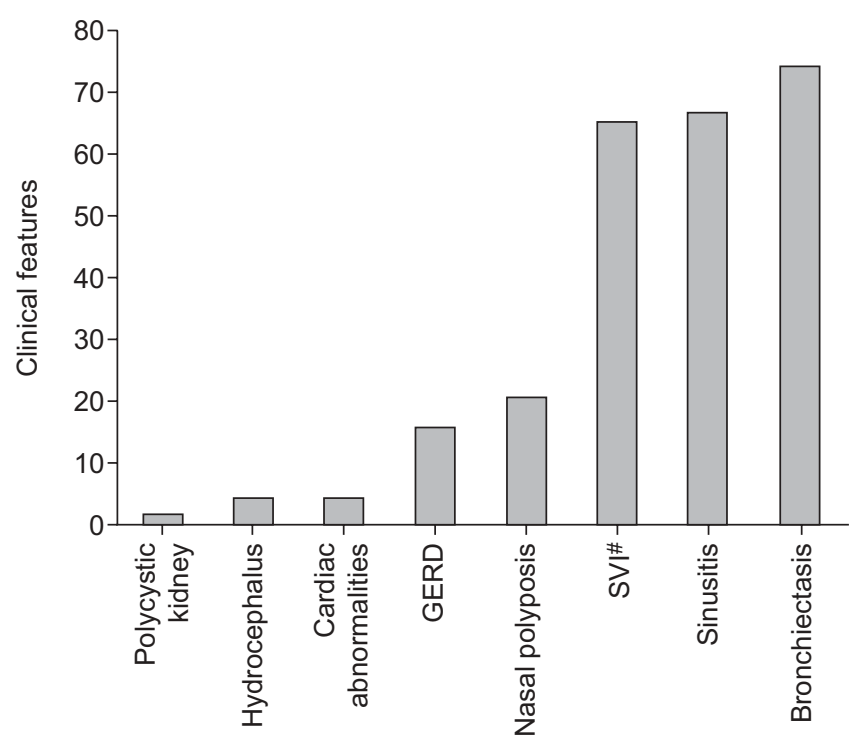

FIGURE 1. Prevalence of different clinical features in the study population GERD: gastro-oesophageal reflux disease; SVI: situs viscerum inversus. ${ }^{\#}$ : one patient had dextrocardia alone. 


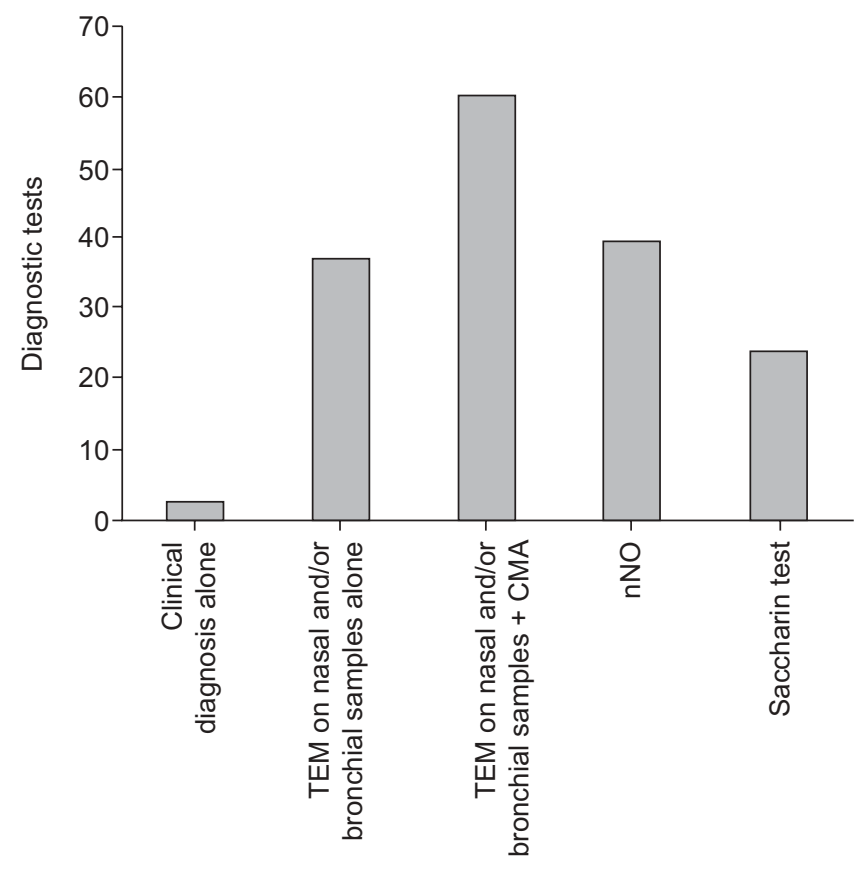

FIGURE 2. Prevalence of diagnostic tests performed in the study population. TEM: transmission electron microscopy; CMA: ciliary motion analysis; nNO: nasal nitric oxide.

evaluation, were only irregularly performed during follow-up (table 2).

\section{Genetic counselling and family education}

PCD was present in other family members of $17(21.8 \%)$ respondents. One $(1.3 \%)$ male adult was a current smoker $\left(10\right.$ cigarettes $\cdot$ day $\left.^{-1}\right)$, and $18(23.1 \%)$ patients were exposed to passive smoke in their home.

\section{Treatments}

Treatment modalities are reported in table 3. Most patients were currently using physiotherapy and breathing exercises/ techniques, as well as were taking regular antibiotics and bronchodilators. In general there was no relationship between time since diagnosis and any treatment except for daily physiotherapy which was less frequently performed by patients who had been diagnosed longer ago $(p=0.04)$. This held true in the adults considered separately $(\mathrm{p}=0.037)$, but not in subjects aged $<18$ yrs regardless of whether the seven youngest patients were included or not. Furthermore, patients performing daily physiotherapy, as well as those on antibiotic therapy, had significantly worse scores both for SGRQ and SF36 than those not following these regimens: significant differences between patients with and without physiotherapy for activity $(\mathrm{p}=0.002)$ and PCS $(\mathrm{p}=0.01)$ and between patients with and without antibiotic treatment for symptoms $(p=0.003)$, impacts $(p=0.003)$, activity $(p=0.002)$ and PCS $(p=0.002)$. Moreover, in the older group there was a progressive reduction in the regular use of antibiotics $(p=0.040)$ and bronchodilators $(\mathrm{p}=0.035)$.

The interpretation of these results is difficult. Longitudinal data are needed to assess whether patients consider treatment a preventive strategy or only a reactive strategy. From this

\begin{tabular}{llc} 
TABLE 2 & Diagnostic procedures performed in follow-up \\
& $\begin{array}{c}\text { Patients with at least one } \\
\text { evaluation }\end{array}$ & $\begin{array}{c}\text { Time between two } \\
\text { evaluations }\end{array}$ \\
\hline Thorax HRCT & $69(88.5)$ & $2.1 \pm 1.3 \mathrm{yrs}$ \\
Paranasal sinuses CT & $48(61.5)$ & $3.3 \pm 2.4 \mathrm{yrs}$ \\
Flow/volume curve & $71(91.0)$ & $23.5 \pm 23.3$ weeks \\
Body plethysmography & $21(26.9)$ & $22.7 \pm 22.5$ weeks \\
Sputum culture & $71(91.0)$ & $21.9 \pm 30.9$ weeks \\
Audiometric test & $55(70.5)$ & $78.1 \pm 72.1$ weeks \\
\hline
\end{tabular}

Data are presented as $n(\%)$ or mean \pm SD. CT: computed tomography; HRCT: high-resolution CT

cross-sectional study we are not able to determine whether these are the sickest patients and this is why they are prescribed a considerable number of treatments, or whether it is the burden of treatment rather than the disease that leads to a poor quality of life. The surgical procedures which were performed are reported in table 4 .

\section{Quality of life after diagnosis of PCD}

A clear majority of patients $(71.8 \%)$ considered their quality of life significantly or slightly improved after diagnosis $(38.5 \%$ and $33.3 \%$, respectively), while only three $(3.7 \%)$ recently diagnosed subjects perceived a worsening in their quality of life. After scoring the answers to the "Quality of life after diagnosis" question (1=greatly worsened to $5=$ greatly improved), there was a statistically significant positive correlation between time since diagnosis and improvement in perceived quality of life $(p=0.02)$, but this was not correlated with age or age at diagnosis.

\section{DISCUSSION}

We report for the first time that a delay in diagnosis of PCD is associated with a poorer subsequent quality of life. This finding is counter-intuitive, since it would be expected that milder cases would be diagnosed later and have a better quality of life. However, it is also possible that children and parents who had been given the diagnosis in early childhood may have adapted better than those diagnosed later. Furthermore, for the majority, quality of life improved after diagnosis; a similar finding has been reported in CF. This adds weight to the evidence that early diagnosis of PCD is beneficial.

It could be argued that the study would have been stronger if a disease-specific questionnaire for PCD patients had been used, and certainly the development of such an instrument is worth further work [16]. However, the use of current instruments allows comparison with previous work. Only one study evaluated quality of life in CF patients with SGRQ [17] and reported that SGRQ scores were higher (indicating poorer quality of life) among patients with $C F$ than in the general population or among patients with chronic obstructive pulmonary disease. Furthermore, the score for age, symptoms, activity and impacts were no different from those observed in our patients with PCD. Another study used the SF-36 [18] to document that pulmonary exacerbations affect physical HRQoL more than psychosocial HRQoL with baseline scores 
TABLE 3 Treatments used by primary ciliary dyskinesia patients

\begin{tabular}{|c|c|c|c|c|c|c|}
\hline & \multicolumn{3}{|c|}{ Usage } & \multicolumn{3}{|c|}{ Correlation of usage } \\
\hline & Never used & In the past & At present & Age yrs & Age at diagnosis yrs & Time since diagnosis yrs \\
\hline Daily physiotherapy & $10(12.82)$ & $10(12.82)$ & $58(74.36)$ & -0.125 (NS) & 0.097 (NS) & $-0.230(0.04)$ \\
\hline Regular antibiotics & $9(11.54)$ & $13(16.67)$ & $56(71.79)$ & 0.147 (NS) & 0.186 (NS) & -0.003 (NS) \\
\hline Regular bronchodilators & $10(12.82)$ & $14(17.95)$ & $54(69.23)$ & -0.069 (NS) & 0.107 (NS) & -0.176 (NS) \\
\hline
\end{tabular}

Data are presented as $n(\%)$, for usage and r-value (p-value) for correlation of usage. Ns: not significant. ${ }^{*}$ : $\mathrm{N}$-acetylcysteine.

being significantly worse $(p=0.03)$ than those observed in our patients but with a mental score $(\mathrm{p}=0.332)$ similar to those observed in our study population.

In our study, as in other chronic lung conditions, respiratory symptoms were assessed by the SGRQ [11, 12], while the impact of the disease on a patient's daily life was measured by version 2 of the SF-36 questionnaire, which is a widely used generic instrument for assessing mental and physical functioning $[13,15]$. Neither the SGRQ or SF-36 questionnaires are validated in children, but they have been previously used in children aged >6-12 yrs [19-21]. SGRQ and SF-36 are complex instruments and are definitely valid if completed by the individual being studied and without the help of others; for this reason subgroup analyses with and without the children who needed help completing the questionnaire were performed. In fact, most children completed the questionnaire unassisted. When we only looked at data from adults, the group in which SGRQ and SF-36 are validated, some correlations are lost, in particular those related to the age of the patients and the time since diagnosis. We cannot determine on the present dataset whether this is due to loss of power because the numbers are smaller, or because the children's data was less reliable because the instruments are not valid; we believe the first to be more likely. It is also possible that as time passes, older patients become resigned to the disease and attitudes change. We found good agreement between the results obtained with a respiratory-specific instrument, such as the SGRQ, and those gained with a generic instrument, i.e. the SF-36, and this is a good indicator both of the specificity of the information and of their general validity. Furthermore, almost

\begin{tabular}{ll}
\hline TABLE 4 & $\begin{array}{l}\text { Incidence of surgery in primary ciliary dyskinesia } \\
\text { patients }\end{array}$ \\
\hline Surgical procedures & Incidence $\mathbf{n}(\%)$ \\
\hline Nasal polypectomy & $8(10.3)$ \\
Lobectomy & $7(8.9)$ \\
Adenotonsillectomy & $7(8.9)$ \\
Tympanostomy tube insertion & $4(5.1)$ \\
Cardiac surgery & $3(3.8)$ \\
Ventriculoperitoneal shunt & $3(3.8)$ \\
Lung transplant & $1(1.3)$ \\
\hline
\end{tabular}

all patients diagnosed by tertiary centres participated in the survey, despite the poor response rates in other postal studies [22]. Thus the sample can be considered representative of the general population of patients with $\mathrm{PCD}$, or at least those being seen in a tertiary centre. What we are not able to determine is whether our findings apply to PCD patients seen in general respiratory clinics, where expertise in the treatment of the condition may be less.

The remarkably high-response rate by the patients is unfortunately not associated with good adherence to the different treatment modalities and this is particularly true in adults. It is clear that current educational programmes are insufficient and that other means of changing behaviour need to be implemented [23]; this represents an important unmet need of those patients.

As expected, evaluating all the patients together, the symptoms, activity and impacts scores of SGRQ increased significantly with age, as seen in healthy subjects. The correlation with age is lost in the separate evaluation of adults; again this may be due to loss of statistical power or it may be a genuine finding. In fact, productive cough, breathlessness and the presence of bronchiectasis were reported with increasing frequency in older patients, considering the group as a whole. This age effect was lost in the adult subgroup, perhaps because almost all of them had marked respiratory compromise. Even though the three sub-scales of the SGRQ provide a detailed picture of respiratory HRQoL, the wide variance in overall health status is better described by the SF-36 PCS and MCS. These scores also have the advantage of well-described Italian population normals $[13,15]$. This allowed us to show that the expected decline of PCS and MCS scores (and also of each subscale) with age was significantly higher in our PCD patients. This early decline was not observed by McMANUs et al. [7] who found little abnormality in standard measures of SF36 during childhood and adolescence. Furthermore they reported that scores were stable after diagnosis. Again this was not observed in our study population who showed a progressive worsening of the disease over time. In our study population it seems that deterioration mainly occurs prior to and during adolescence, as after the age of $18 \mathrm{yrs}$ much of the correlation with age is lost. There are a number of possible reasons for this which need to be investigated further; these include an adolescent rebellion and refusal to perform treatment, adolescent risk-taking behaviour, such as smoking or substance abuse, and change of pattern of care to a possibly less specialist adult unit. 
The causes for these discrepancies are not known. One could speculate that, in the UK, any or all of earlier diagnosis, more aggressive early treatment or better adherence to treatment might be possible explanations. In this regard, the age of diagnosis in the current population (9.4 yrs) was significantly later than in the UK population reported by COREN et al. [24], which was 4.4 yrs. Another possibility could be better continuity between paediatric and adult care, something which is not yet well structured in Italy. However, longitudinal analysis is the only way to resolve these issues, and international studies on the topic should be encouraged. McMANus et al. [7] reported a continual decline with age (1.5 SD below the population mean) on the physical scores of the SF36 reflecting a moderate degree of morbidity on normal physical functioning which is progressive across the lifespan; this is partially confirmed by our study. However, McMANUS et al. [7] did not show a significant effect of time since diagnosis either on the MCS and PCS of the SF-36 questionnaire or on the symptoms and activity scores of the SGRQ.

Early identification of the disease is an important unmet need of these patients. This is particularly evident in female patients, since Yentl syndrome (sex bias towards diagnostic procedures and treatment), as described for asthma [25], also appears to be present in PCD. However, it is reassuring to observe that the sex bias observed in relation to diagnosis is not found in relation to care since no difference between female and male patients is reported in scores of SGRQ and SF-36 questionnaires.

In addition to previously used instruments, we used a novel questionnaire on PCD/Kartagener Syndrome, the first specific and structured questionnaire on this disease. This provides more information on PCD than the original questionnaire by MCMANUs et al. [7], which only assessed presence of situs inversus, family history, smoking and treatments.

In this regard, our study demonstrates that the prevalence of cardiac defects is similar to that recently reported in the general population when transthoracic echocardiography was performed [26]. This may reflect the number of diagnostic procedures which are requested in patients with unexplained respiratory symptoms, particularly in subjects with situs solitus. In our study population cardiac defects were discovered prior to the diagnosis of PCD.

The classical diagnostic tests required for an appropriate diagnosis, such as ciliary beat frequency and pattern, and transmission electron microscopy, were not performed in all the patients. This underlines another unmet need, namely the right of each patient to be properly diagnosed in tertiary centres. Furthermore, follow-up procedures such as audiometry, sputum culture and lung function evaluations were not performed in some patients. Audiometry is essential in paediatric patients since hearing loss is found in up $25 \%$ of children with PCD [24] and sputum culture and regular spirometry should be part of the routine follow-up of chronic inflammatory airways diseases [4, 5]. High-resolution computed tomography (HRCT) of the lungs and of paranasal sinuses were not performed in $12 \%$ and $40 \%$ of the patients, respectively, especially in the younger ones. Chest HRCT is used to define the extent of bronchiectasis and can be used to monitor progression of the disease; however, there is little evidence that regular computed tomography scans affect outcome in PCD and the potential high lifetime cumulative radiation exposure should be considered [1].

High-quality genetic counselling is another priority to ensure that parents clearly understand and consider the risks of the same disease in subsequent pregnancies in the context of a complex condition where multiple genes are involved and where prenatal screening is usually not possible. Obviously an early diagnosis is essential for counselling since late diagnosis may preclude planning of further pregnancies. Education of the family is a further important concern, since one out of five patients was exposed to passive smoking in their home. This prevalence is lower than that reported in general in the Italian population [27], but it cannot be considered satisfactory. Considering all patients together, $>10 \%$ of the patients never used daily physiotherapy and $>25 \%$ were not currently using airway clearance techniques in their daily management at the time of the survey. The figures for those aged $\geqslant 18$ yrs were $12 \%$ and $28 \%$, respectively, but daily physiotherapy was reportedly performed in $79 \%$ of patients aged $<14$ yrs. This lack of utilisation of airway clearance was despite intensive education from tertiary care centres. The level of compliance with physiotherapy was the same whether patients were recruited from tertiary centres or responded through the website. Thus, another unmet need for patients and parents is to understand that physiotherapy should be performed on a daily basis and increased during infective episodes, since antibiotics alone should not be relied upon. The ways by which to modify behaviour should be studied and ideally implemented immediately after the diagnosis, as well as during follow-up of the patients, especially since there is clear deterioration in treatment adherence in patients aged $>18$ yrs $[23,28]$. Compliance with physiotherapy decreases with time, probably reflecting the fact that patients progressively give up and passively accept their disease, leading to treatment (both physiotherapy and antibiotics) being reactive to symptoms rather than as prevention of deterioration. Thus another unmet need is the necessity of continuous physical and psychological support.

The physical care of the PCD patient is important, but the psychological effects on both the family and the patient must also be considered. It is interesting to note that a few recently diagnosed patients describe worsening of quality of life immediately after diagnosis and this probably represents a transitory emotional distress which is often seen as an understandable reaction to a severe illness. Therefore, it is important that the specialist team working together with family physicians evaluate whether the initial reaction of anger and denial is replaced by acceptance and coping with the proper management of the disease [29].

In conclusion, better education of general physicians and paediatricians on the disease is essential; once PCD patients are diagnosed they need integrated management between specialised centres, district hospitals and family doctors. This is expected to increase shared knowledge, decrease mistrust of general practitioner who is generally poorly informed of what is a rare disease, and possibly to facilitate early diagnosis [30]. There is also the need for support from peer groups and volunteers to diminish stigmatisation and to give practical help to patients with PCD and to their families. 


\section{STATEMENT OF INTEREST}

Statement of interest for A. Bush and A.L. Boner can be found at www. erj.ersjournals.com/misc/statements.dtl

\section{ACKNOWLEDGEMENTS}

We are grateful to Unione Industriale Pisana (Pisa, Italy) for supplying the scientific instruments.

\section{REFERENCES}

1 Bush A, Chodhari R, Collins N, et al. Primary ciliary dyskinesia: current state of the art. Arch Dis Child 2007; 92: 1136-1140.

2 Schidlow DV. Primary ciliary dyskinesia (the immotile cilia syndrome). Ann Allergy 1994; 73: 457-468.

3 Noone PG, Leigh MW, Sannuti A, et al. Primary ciliary dyskinesia: diagnostic and phenotypic features. Am J Respir Crit Care Med 2004; 169: 459-467.

4 Ellerman A, Bisgaard H. Longitudinal study of lung function in a cohort of primary ciliary dyskinesia. Eur Respir J 1997; 10: 2376-2379.

5 Hellinckx J, Demedts M, De Boeck K. Primary ciliary dyskinesia: evolution of pulmonary function. Eur J Pediatr 1998; 157: 422-426.

6 Marshall WF. The cell biological basis of ciliary disease. J Cell Biol 2008; 180: 17-21.

7 McManus IC, Mitchison HM, Chung EM, et al. Primary ciliary dyskinesia (Siewert's/Kartagener's syndrome): respiratory symptoms and psycho-social impact. BMC Pulm Med 2003; 3: 4.

8 Juniper EF, Guyatt GH. Development and testing of a new measure of health status for clinical trials in rhinoconjunctivitis. Clin Exp Allergy 1990; 21: 77-83.

9 Vuurman EF, van Veggel LM, Uiterwijk MM, et al. Seasonal allergic rhinitis and antihistamine effects on children's learning. Ann Allergy 1993; 71: 121-126.

10 Centro di riferimento per la Diagnosi e la Terapia della Discinesia Ciliare Primaria/Sindrome di Kartagener - Pisa. Available from: www.dcp-pisa.it

11 Wilson $\mathrm{CB}$, Jones PW, O'Leary CJ, et al. Validation of the St. George's Respiratory Questionnaire in bronchiectasis. Am J Respir Crit Care Med 1997; 156: 536-541.

12 Carone M, Bertolotti G, Anchisi F, et al. The St. George's Respiratory Questionnaire (SGRQ): Italian version. Rassegna Patol App Respir 1999; 14: 31-37.

13 Apolone G, Mosconi P. The Italian SF-36 Health Survey: translation, validation and norming. J Clin Epidemiol 1998; 51: 1025-1036.

14 Jones PW, Quirk FH, Baveystock CM. The St. George's Respiratory Questionnaire. Resp Med 1991; 85: 25-31.
15 Apolone G, Mosconi P, Ware J, eds. Questionario Sullo Stato Di Salute SF-36. Manuale d'uso e Guida all'interpretazione dei risultati. Milan, Guerini Ed associati, 1997.

16 Henry B, Aussage P, Grosskopf C, et al. Development of the Cystic Fibrosis Questionnaire (CFQ) for assessing quality of life in pediatric and adult patients. Qual Life Res 2003; 12: 63-76.

17 Padilla A, Olveira G, Olveira C, et al. Validity and reliability of the St George's Respiratory Questionnaire in adults with cystic fibrosis. Arch Bronconeumol 2007; 43: 205-211.

18 Yi MS, Tsevat J, Wilmott RW, et al. The impact of treatment of pulmonary exacerbations on the health-related quality of life of patients with cystic fibrosis: does hospitalization make a difference? J Pediatr 2004; 144: 711-718.

19 Jones PW. Quality of life, symptoms and pulmonary function in asthma: long-term treatment with nedocromil sodium examined in a controlled multicentre trial. Nedocromil Sodium Quality of Life Study Group. Eur Respir J 1994; 7: 55-62.

20 Sheridan RL, Hinson MI, Liang MH, et al. Long-term outcome of children surviving massive burns. JAMA 2000; 283: 69-73.

21 Dawson J, Fitzpatrick R, Carr A. The assessment of shoulder instability. The development and validation of a questionnaire. J Bone Joint Surg Br 1999; 81: 420-426.

22 Nakash RA, Hutton JL, Jørstad-Stein EC, et al. Maximising response to postal questionnaires - a systematic review of randomised trials in health research. BMC Med Res Methodol 2006; 6: 5 .

23 Huffman M. Health coaching: a new and exciting technique to enhance patient self-management and improve outcomes. Home Healthc Nurse 2007; 25: 271-274.

24 Coren ME, Meeks M, Morrison I, et al. Primary ciliary dyskinesia: age at diagnosis and symptom history. Acta Paediatr 2002; 91: 667-669.

25 Healy B. The Yentl syndrome. N Engl J Med 1991; 325: 274-276.

26 Gupta-Malhotra M, Dave A, Sturhan BC, et al. Prevalence of undiagnosed congenital cardiac defects in older children. Cardiol Young 2008; 18: 392-396.

27 Ronchetti R, Bonci E, Martinez FD. Passive smoking in childhoodtobacco smoke. Lung 1990; 168: Suppl, 313-319.

28 Sevick MA, Trauth JM, Ling BS, et al. Patients with complex chronic diseases: perspectives on supporting self-management. J Gen Intern Med 2007; 22: Suppl. 3, 438-444.

29 Veale BM. Meeting the challenge of chronic illness in general practice. Med J Aust 2003; 179: 247-249.

30 Whalley S, McManus IC. Living with primary ciliary dyskinesia: a prospective qualitative study of knowledge sharing, symptom concealment, embarrassment, mistrust, and stigma. BMC Pulm Med 2006; 6: 2. 